Ambient Science, 2019: Vol. 06(Sp1); 41-42

DOI:10.21276/ambi.2019.06.sp1.nn02

\title{
Therapeutic Potential of the Cannabis plant: Application and
} Knowledge Gaps

\section{Jyoti Chawla}

Department of Chemistry, Manav Rachna International Institute of Research Studies, Sector-43, Surajkund Road, Faridabad, Haryana, India

\section{Introduction:}

Cannabis is cultivated by human beings since ancient times. Characteristics and quality of Cannabis plants depend on different factors such as type of soil, use of pesticides, herbicides, temperature conditions, degree and type of contamination, etc. In the cannabis plant, most important cannabinoids are cannabidiol (CBD) and tetrahydrocannabinol (THC). The concentration of THC is normally found to be highest than $\mathrm{CBD}$ and it may be mixed in the preparation as per requirement. Mechoulam \& Shvo (1963) isolated the cannabidiol (CBD) and tetrahydrocannabinol (THC) from the cannabis plant. The attempt to invigorate cannabis as a medicine got motivated due to its therapeutic potential for many diseases including cancer, Crohn's disease, Sclerosis, Alzheimer's disease, etc. Cannabidiol is a non-psychotropic component of cannabis. Various studies have confirmed the antipsychotic, antiinflammatory, and anticonvulsant properties of CBD (Mechoulam et al., 2002). Preclinical studies have also revealed the positive effects of $\mathrm{CBD}$ for diseases including diabetes, nerve cell disease, cardiovascular and cancer (Stanley et al., 2013; Walsh et al., 2010; Lacson et al., 2012). However, the risks of misuse, poisoning, and other harmful consequences of consumption of these compounds led to the implementation of preventive laws for prohibiting the growth, ownership, and consumption of cannabis in many regions across the world. Few knowledge gaps regarding the risk and benef its of THC and CBD are still exist. The aim of the paper is to discuss the reported preclinical, clinical, and epidemiological studies on assessing the therapeutic potential of the cannabis plant. The associated biomarkers, regulatory and legal aspects have also been discussed.

Effect of cannabidiol \& tetrahydrocannabinol: Researchers made several important discoveries regarding the effects of cannabis including where and how cannabis works in the brain via the endocannabinoid system.

Epidemiologic studies confirm that cannabis use can supplement the risk of severe mental disorders (Gage et al., 2016). Nicholson et al. (2004) observed the effect of CBD

\author{
Key words: Cannabidiol, Tetrahydrocannabinol, \\ Cancer, Medicinal Plants
}

and THC on 21-34 years old healthy males as well as females. Four treatments formulated in ethanol to propylene glycol (50:50) including $5 \mathrm{mg}$ CBD and THC, $25 \mathrm{mg}$ CBD and THC, THC $15 \mathrm{mg}$ and dummy pill were given using an or mucosal spray (for $30 \mathrm{~min}$ in the night) to study early morning behavior and sleep. Signif icant dose-response relationship was conf irmed for 1:1 combination of THC/CBD in males as well as in females and more sleepiness and alteration in behavior was conf irmed for the high dose. However, with 15 mg THC on next day memory of subjects impaired. Jadoon et al. (2017) studied the effect of CBD (6oo mg) on blood pressure of nine healthy male volunteers. CBD reduced resting systolic blood pressure and stroke volume, with unchanged cardiac output and increase heart rate. In vitro studies have shown that CBD can be converted to THC with prolonged exposure to simulated gastric fluid. However, this may not be representative of the results in vivo and caution should be taken while extrapolating in vitro effects/results to the in vivo scenario. However, the majority of in vivo animal studies indicated that CBD is capable of decreasing tumor growth in an effective way.

\section{Legal implications and research:}

The vulnerability of misuse, poisoning, and related harmful effects due to the consumption of these compounds led to the implementation of preventive laws for prohibiting the growth, ownership and consumption of cannabis in many regions across the world. Uruguay, a South American country was the first one to legalize the regulated sale and use of cannabis but as per law, foreigners are not allowed to buy cannabis from Uruguay. However, Uttarakhand legalized the farming of cannabis in 2015 for only industrial purposes. Union Government has also issued the license to the Council of Scientific and Industrial Research in 2017 to cultivate cannabis for research work in association with the Bombay Hemp Company. In February 2018, Patanjali Ayurveda has begun research on cannabis and its extracts for its use in medicines. On 25th November, 2018; results of the first clinical study on cannabis were published in India by Central Council for Research in Ayurvedic Sciences. The

*Corresponding Author: rajeshghangal.fet@mriu.edu.in 
work was focused on the utilization of cannabis for cancer patients as a drug for improving strength and health.

\section{Some findings and knowledge gaps:}

Morell et al. (2016) found a remarkable decrease in cannabinoid receptors during neuroendocrine differentiation that is a functional trait of prostate cancer. Cooper \& Haney (2016) compared the analgesic effect of cannabis with 3.5-5.6\% THC or no THC on both females and males and found that cannabis with 3.5-5.6 \% THC has more analgesic effect on males but failed to decrease pain in females. It was further highlighted that there was a need for further investigation to further explore the genderdependent responses in cannabis's analgesic effects. Ladino et al. (2014) in a series case study of 18 common profile found that epilepsy patients who were using cannabis as a drug before the prescription was effective for seizure control. The study, however, was unable to analyze the actual effect of cannabis due to the simultaneous use of other medications by patients. A recent study on 81 years old patient with lung adenocarcinoma indicated that CBD led to partial tumor response after self-administration of CBD oil for a month (Sule-Suso et al., 2019). Another Clinical study including cannabinoid treatment showed positive effects on Glioblastoma patients (Dumitru et al., 2018). Although research evidence of preclinical and clinical studies is not sufficient to confirm the use of cannabis for various health issues, some important key findings are mentioned in Fig.-1.

The majority of results from different in vivo animal studies indicate that cannabinoids can effectively decrease tumor invasion \& growth. The studies reviewed to conf irm that cannabis-based drugs may lessen pain and other associated symptoms post- chemo and radiotherapy in cancer patients. Moreover, clinical studies that can estimate the cannabinoid effect in the human body are inadequate; however, these studies explained the safety and effectiveness of cannabis as anti-neoplastic agents. Large scale clinical trials are required to inspect the activity of cannabis in clinically translatable animal models for further evaluation and legal usage of cannabis as medicine.

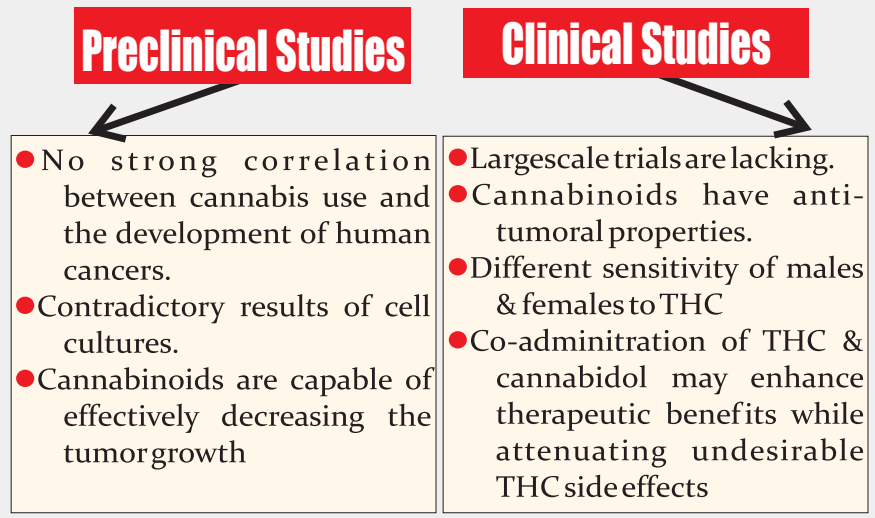

Figure-1: A comparative chart between old and new experiences

\section{References:}

Cooper, Z.D. \& Haney, M. (2016): Sex-dependent effects of cannabis-induced analgesia. Drug Alco. Dep., 167:112-120.

Dumitru, C.A., Sandalcioglu, I.E. \& Karsak, M. (2018): Cannabinoids in glioblastoma therapy: new applications for old drugs. Front Mol. Neurosci., 11:159-157.

Gage, S.H., Hickman, M. \& Zammit, S. (2016): Association between cannabis and psychosis: epidemiologic evidence. Biol. Psychiatry., 79(7):549-556.

Jadoon, K.A., Tan, G.A., Saoirse E. O'Sullivan. (2017): A single dose of cannabidiol reduces blood pressure in healthy volunteers in a randomized crossover study. LCI Insight., 2(12)

Lacson, J.C., Carroll, J.D., Tuazon, E., Castelao, E.J., Bernstein, L. \& Cortessis, V.K. (2012): Population-based case-control study of recreational drug use and testis cancer risk confirms an association between cannabis use and nonseminoma risk. Cancer, 118(21):5374-5383.

Ladino, L.D., Hernández-Ronquillo, L. \& Tellez-Zenteno, J.F. (2014): Medicinal Marijuana for Epilepsy: A Case Series Study. CanadianJ. Neurol. Sci., 41(6)753-758.

Mechoulam, R., Parker, L.A. \& Gallily, R. (2002): Cannabidiol: an overview of some pharmacological aspects.J. Clin. Pharmacol., 42(S1):11S-19S.

Mechoulam, R. \& Shvo, Y. (1963): Hashish I. The structure of cannabidiol. Tetrahedron., 19(12):2073-2078.

Morell, C., Bort, A., Vara, D., Ramos-Torres, A., RodríguezHenche, N. \& Díaz-Laviada, I. (2016): The cannabinoid WIN 55,212-2 prevents neuroendocrine differentiation of LNCaP prostate cancer cells. Prost. Can. Prost. Dis., 19(3):248-257.

Nicholson, A.N., Turner, C., Stone, B.M. \& Robson, P.J. (2004): Effect of $\Delta^{9}$-tetrahydrocannabinol and cannabidiol on nocturnal sleep and early-morning behavior in young adults. J. Clin. Psychopharmacol., 24(3):305-313.

Stanley, C.P., Hind, W.H., O'Sullivan, S.E. (2013): Is the cardiovascular system a therapeutic target for cannabidiol? Br.J. Clin. Pharmacol., 75(2):313-322.

Sule-Suso, J., Watson, N. A., van Pittius, D. G. \& Jegannathen, A. (2019): Striking lung cancer response to self-administration of cannabidiol: A case report and literature review. $\underline{S A G E}$ Open Medical Case Reports, 7:2050313X19832160.

Walsh, S.K., Hepburn, C.Y., Kane, K.A. \& Wainwright, C.L. (2010): Acute administration of cannabidiol in vivo suppresses ischaemia-induced cardiac arrhythmias, and reduces infarct size when given at reperfusion. 